\title{
APPLICATION OF NUMERICAL INVERSE METHOD IN CALCULATION OF COMPOSITION-DEPENDENT INTERDIFFUSION COEFFICIENTS IN FINITE DIFFUSION COUPLES
}

\author{
Yuanrong Liu ${ }^{l, 2}$, Weimin Chen ${ }^{1}$, Jing Zhong ${ }^{1}$, Ming Chen ${ }^{3}$, Lijun Zhang ${ }^{1 *}$ \\ ${ }^{1}$ Science and Technology on High Strength Structural Materials \\ Laboratory, Central South University, Changsha, P.R. China \\ ${ }^{2}$ AECC Industrial Co., Ltd., Zhuzhou, P.R. China \\ ${ }^{3}$ Department of Energy Conversion and Storage, Technical University \\ of Denmark, Roskilde, Denmark
}

Received 28.07.2017

Accepted 23.08.2017

\begin{abstract}
The previously developed numerical inverse method was applied to determine the composition-dependent interdiffusion coefficients in single-phase finite diffusion couples. The numerical inverse method was first validated in a fictitious binary finite diffusion couple by pre-assuming four standard sets of interdiffusion coefficients. After that, the numerical inverse method was then adopted in a ternary $\mathrm{Al}-\mathrm{Cu}-\mathrm{Ni}$ finite diffusion couple. Based on the measured composition profiles, the ternary interdiffusion coefficients along the entire diffusion path of the target ternary diffusion couple were obtained by using the numerical inverse approach. The comprehensive comparison between the computational diffusion properties and the experimental data indicates that the numerical inverse method is also applicable to the high-throughput determination of the composition-dependent interdiffusion coefficients in finite diffusion couples.
\end{abstract}

Keywords: Diffusion; Interdiffusion coefficient; Numerical inverse method; Finite diffusion couple.

\footnotetext{
* Corresponding author: Lijun Zhang, lijun.zhang@csu.edu.cn
} 


\section{Introduction}

High-throughput measurement of accurate interdiffusion coefficients is of great significance for diffusion and kinetic studies in various materials processes [1,2]. The solid semi-infinite diffusion couple is the frequently used experimental technique for measurement of interdiffusion coefficients in the target phase. Numerous investigations on the measurement of interdiffusion coefficients by using the semi-infinite diffusion couple together with either the traditional Bolzmann-Matano method and its variants [35] for binary systems or the Matano-Kirkaldy method [6] for ternary systems can be found in the literature [2,7-12]. Sometimes, when the total thickness of the diffusion couple is not large enough or the diffusion time is too long, diffusion distance may exceed the entire domain. In this case, the predefined semi-infinite diffusion couple turns out to be a finite one. Moreover, the finite diffusion couple is very common in the coating technology. However, different from the semi-infinite diffusion couple, such finite diffusion couple has seldom been employed to measure the interdiffusion coefficients in the target phase.

One of the obstacles for measuring interdiffusion coefficients via the finite diffusion couple lies in its complex nature. For instance, in a binary finite diffusion couple, the terminal compositions at the boundaries deviate from the nominal ones. Figure 1 presents a typical composition profile of a solute element in a fictitious binary finite diffusion couple after a certain diffusion time. If one utilizes the traditional BoltzmannMatano method to evaluate the interdiffusion coefficients in such diffusion couple, the accurate calculation of interdiffusion flux at the distance $X^{\prime}$ is the prerequisite. As shown in Fig. 1, the time-integrated interdiffusion flux at the distance $X^{\prime}$ in the finite diffusion couple should be the summation of area A and area B, which is different from the semiinfinite diffusion couple. Such extra calculations will make the cases much more complex in ternary and higher order systems, especially with the asymmetric composition profiles or with the up-hill diffusion phenomenon.

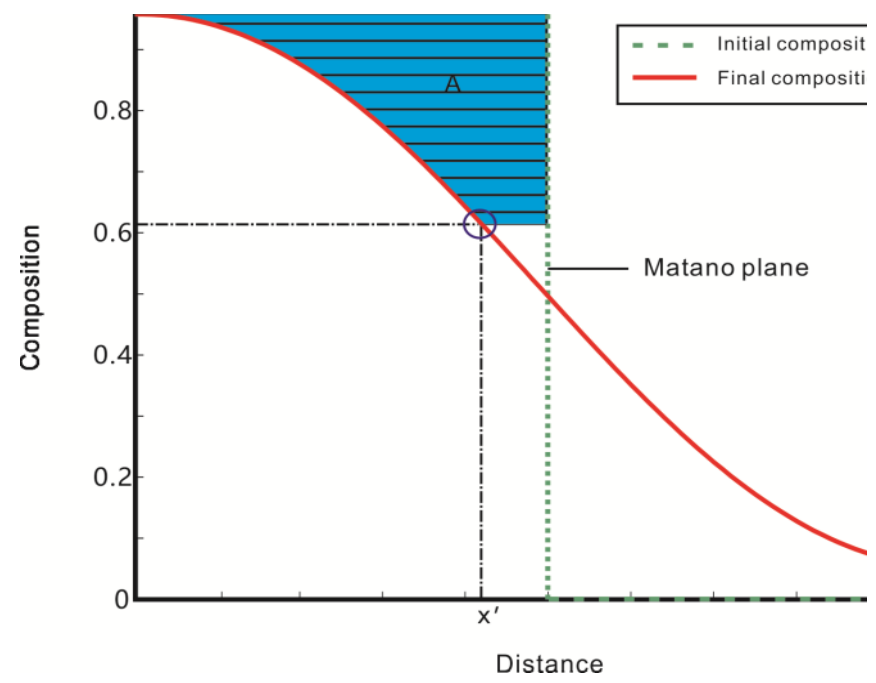

Fig. 1. Schematic diagram for determination of interdiffusion flux in a finite diffusion couple via the traditional Boltzmann-Matano method. 
Recently, a pragmatic numerical inverse method was proposed for high-throughput determination of the composition-dependent interdiffusion coefficients by using a single diffusion couple [13] and then successfully applied to several different alloys [13-18]. Very recently, such a pragmatic numerical inverse method has been augmented and demonstrated to be applicable in binary, ternary and even multicomponent systems $[19,20]$. One of the advanced features of the pragmatic numerical inverse method lies in that it can provide abundant interdiffusion coefficients, which can meet the requirement of MGI (Materials Genome Initiative) and ICME (Integrated Computational Materials Engineering) projects well. While the other advanced feature is its simple input, including only the initial compositions for the couples, annealing conditions, and boundary conditions. Moreover, the calculation of interdiffusion flux is excluded in this method. Thus, the pragmatic numerical inverse method is anticipated to serve as an effective way to evaluate the interdiffusion coefficients in finite diffusion couples.

Consequently, the major purpose of the present work is to apply the pragmatic numerical inverse method to efficiently measure the composition-dependent interdiffusion coefficients in finite diffusion couples. In the first part, the pragmatic numerical inverse method was validated in different binary cases. The strategy proposed by Ahmed et al. [21] was adopted in the present work, i.e., that four standard types of interdiffusion coefficients (including constant, linear, quadratic composition dependence of interdiffusion coefficients) were set in advance, and then used to generate different composition profiles for a binary finite diffusion couple with the same initial and annealing conditions. In order to mimic the "scattered" experimental data in real cases, the Gaussian-distributed noise was added in. The pre-assumed interdiffusion coefficients can be regarded as the criteria to validate the numerical inverse method. In the second part, the application of the pragmatic numerical inverse method in a real case of the ternary Al-Cu-Ni system was demonstrated.

\section{Numerical inverse method}

In a hypothetical A-B-C ternary system, in which A and B are solutes while $\mathrm{C}$ is chosen as the solvent, Fick's second law can be given as

$$
\frac{\partial c_{i}}{\partial t}=\frac{\partial}{\partial X}\left(\tilde{D}_{i A}^{C} \frac{\partial c_{A}}{\partial X}+\tilde{D}_{i B}^{C} \frac{\partial c_{B}}{\partial X}\right)(i=A \text { or } B)
$$

where $c_{i}$ is the composition of component $i, X$ is the distance, and $t$ is the diffusion time. $\tilde{D}_{A A}^{C}$ and $\tilde{D}_{B B}^{C}$ are the two main interdiffusion coefficients, while $\tilde{D}_{A B}^{C}$ and $\tilde{D}_{B A}^{C}$ are the two cross interdiffusion coefficients. Taking the vacancy flow effect into consideration, interdiffusion coefficient $\tilde{D}_{i j}^{C}$ and the mobility $M_{i}$ can be related via $[13,22]$

$$
\begin{gathered}
\tilde{D}_{i j}^{C}=R T\left[M_{i} \Phi_{i j}^{C}-c_{i}\left(M_{A} \Phi_{A j}^{C}+M_{B} \Phi_{B j}^{C}+M_{C} \Phi_{C j}^{C}\right)\right]+ \\
S\left[\left(M_{i}-c_{A} M_{A}-c_{B} M_{B}-c_{C} M_{C}\right) \frac{2 c_{i} R T \sum_{m}\left(M_{m} \Phi_{m j}^{C}\right)}{A_{0} \sum_{m}\left(c_{m} M_{m}\right)}\right]
\end{gathered}
$$


where $R$ is the gas constant and $T$ absolute temperature. Thermodynamic factor $\Phi_{i j}^{C}$ can be expressed as

$$
\Phi_{i j}^{C}=\frac{c_{i}}{R T} \cdot\left(\frac{\partial \mu_{i}}{\partial c_{j}}-\frac{\partial \mu_{i}}{\partial c_{C}}\right)
$$

where $\mu_{i}$ is the chemical potential of component $i$. In general, $\mu_{i}$ can be obtained from the reliable thermodynamic descriptions which are usually available for most alloy systems nowadays. Although reasonable thermodynamic data facilitate the computation of interdiffusivity by the numerical inverse method, it is not a must [19]. That is because the composition profiles are the input and the composition-dependent interdiffusivities are the output in the numerical inverse method. While the thermodynamic factor, as well as the mobility Ms, are the adjustable parameters. Hence, different thermodynamic descriptions can only affect the assessed $M \mathrm{~s}$, but not influence the quality of the obtained interdiffusivities. Alternatively, the thermodynamic factor can be treated as a numerical parameter when the thermodynamic description of the target phase is absent. The second part of Eq. (2) denotes the vacancy-wind effect. The vacancy-wind effect is considered when $s$ equals to 1 , while not when $s$ equals to $0 . A_{0}$ is a factor depending on crystal structure and taken as 7.15 for fcc crystal structure.

As for the atomic mobility in Eq. (2), its formula can follow the work by Andersson and Agren [23]

$$
M_{i}=\frac{1}{R T} \exp \left(\frac{R T \ln M_{i}^{0}-Q_{i}}{R T}\right)=\frac{1}{R T} \exp \left(\frac{\Delta G_{i}}{R T}\right)
$$

where $M_{i}^{0}$ is the frequency factor, and $Q_{i}$ is the activation enthalpy. If the ferromagnetic contribution to diffusion can be neglected, $R \operatorname{TIn} M_{i}^{0}$ and $Q_{i}$, can be merged into one parameter, $\Delta G_{i}$, which can be expanded via the Redlich-Kister polynomial,

$$
\begin{aligned}
& \Delta G_{i}=c_{A} \Delta G_{i}^{A}+c_{B} \Delta G_{i}^{B}+c_{C} \Delta G_{i}^{C}+c_{A} c_{B} \Delta G_{i}^{A, B}+c_{A} c_{C} \Delta G_{i}^{A, C}+ \\
& c_{B} c_{C} \Delta G_{i}^{B, C}+c_{A} c_{B} c_{C} \Delta G_{i}^{A, B, C}
\end{aligned}
$$

Here, $\Delta G_{i}^{A}, \Delta G_{i}^{B}$, and $\Delta G_{i}^{C}$ are the end-members corresponding to self- and impurity diffusivities of component $i$ in elements $\mathrm{A}, \mathrm{B}$, and $\mathrm{C}$, respectively, while $\Delta G_{i}^{A, B}$ $, \Delta G_{i}^{A, C}, \Delta G_{i}^{B, C}$ and $\Delta G_{i}^{A, B, C}$ are the interaction parameters for the mobility of component $i$ in the binary A-B, A-C, B-C, and ternary A-B-C systems. In principle, all the end-members and interaction parameters might be regarded as adjustable parameters in this method. Considering that there are too many adjustable parameters in Eq. (5) for 
the ternary A-B-C system, the end-members are first fixed in practice because these parameters are corresponding to self- and impurity diffusivities, which can be obtained from the experimental measurements, first-principles calculations, molecular dynamic simulation or some semi-empirical relations nowadays. The interaction parameters are then treated as adjustable ones. Integrating Eq. (2) to Eq. (5), the composition-dependent interdiffusion coefficients can be evaluated when the interaction parameters are assessed.

By solving Fick's second law (Eq. (1)) with the finite difference method, the composition profiles of the experimental diffusion couples can be recovered. The error between the measured and the simulated concentration profiles can be defined as

$$
\min \langle\text { error }\rangle=\min \left\langle\frac{1}{N} \sum_{i=1,2} \sum_{j=1}^{N}\left(\left|c_{i j}^{c a l}-c_{i j}^{\exp }\right|\right)\right\rangle
$$

where $c_{i j}^{c a l}$ and $c_{i j}^{\exp }$ are the calculated and the experimental concentrations of component $i$ at the $j$-th point, respectively, and $N$ is the number of the experimental data. By iteratively improving the adjustable parameters with appropriate algorithms, the error in Eq. (6) can be gradually minimized. Once the error is minimized to a certain extent, the optimized adjustable parameters can be taken as the reasonable estimation of the interaction parameters. Consequently, the objective interdiffusivities can be computed with the optimized interaction parameters according to Eqs. (2) to (5).

The genetic algorithm was used for the present optimization of adjustable parameters. This algorithm works in a way that unknowns will gradually evolve into satisfactory solution by repeating a series of evolution operations: mutation, crossover, and selection. Thus, the present genetic algorithm is different from the strictly "standard" method, which always leads to a local minimum, but is able to find satisfactory results for the optimization problem, and may result in the global minima. With the optimal set of fitting parameters, the composition-dependent interdiffusivities in the target ternary system can be computed via Eq. (2). All the obtained ternary interdiffusivities were subject to the check via the following thermodynamically stable constraints [24]:

$$
\begin{gathered}
\tilde{D}_{A A}^{C}+\tilde{D}_{B B}^{C}>0 \\
\tilde{D}_{A A}^{C} \cdot \tilde{D}_{B B}^{C}-\tilde{D}_{A B}^{C} \cdot \tilde{D}_{B A}^{C} \geq 0 \\
\left(\tilde{D}_{A A}^{C}-\tilde{D}_{B B}^{C}\right)^{2}+4 \cdot \tilde{D}_{A B}^{C} \cdot \tilde{D}_{B A}^{C} \geq 0
\end{gathered}
$$

Only the interdiffusivities that fulfilled these constraints were retrieved. This pragmatic numerical inverse method can also be simplified to be applicable to binary systems, and thus can be directly adopted in the present work for determining interdiffusion coefficients in a binary system. It should be noted that if abundant experimental composition profiles over wide composition range at different temperatures are available, the evaluated mobility parameters using the numerical inverse method together with accurate thermodynamic descriptions may be employed to predict the diffusivities at a different temperature. But this is not the major aim of the present approach, which aims at evaluating the composition-dependent interdiffusivities along 
the entire diffusion path of the target single-phase diffusion couple based on only the measured composition profiles.

In fact, the present numerical inverse method is not the first try to extract the composition-dependent interdiffusivity matrices from a single diffusion couple $[25,26]$. For the process that measures interdiffusivity matrices using a single diffusion couple by comparing the measured and the predicted composition profiles, the problems become mathematically ill-posed and the error in interdiffusivity matrices rapidly becomes very large [25-27]; for instance, if the minor eigenvector of interdiffusivity matrices aligns with the composition vector made by the end compositions used in the diffusion couple. In order to avoid this potential problem, the usage of multiple diffusion couples might be one solution according to the comprehensive analysis $[25,27]$. For the binary fictitious cases with the preset interdiffusivities in the present work, the relative error between the standard and the calculated interdiffusivities is a straightforward measure of the quality for the numerical inverse method. While in the real ternary case where no prior information about the analytical solution is known, the uncertainty evaluated via the formalism of the error propagation is appropriate. Taking the entire process of the adjustable parameters as a function $f$, the uncertainties of the evaluated quantities can be evaluated according to the formalism of error propagation [28]

$$
u(f(A, B \ldots))=\sqrt{\sum_{\alpha=A, B . .}(u(\alpha))^{2}\left(\frac{\partial f}{\partial \alpha}\right)^{2}}
$$

Here, $A$ and $B \ldots$ are the correlation quantities of function $f$, while $u(\alpha)$ $(\alpha=A, B \ldots)$ is the uncertainty in the measurements of variable $\alpha$ such as concentration, the position of the Matano plane and so on. During the evaluation of the experimental errors, the uncertainties from different sources that included the experimental measurements and the predicted concentration profiles were propagated first in the calculation of atomic mobility in Eq. (4) and then in the calculation of diffusivities in Eq. (2). To eliminate the effect of the absolute value, the relative error, which is equal to the uncertainty divided by the absolute value of the interdiffusivity, rather than the uncertainty itself, was utilized in the present work. Moreover, considering that one relative error can be determined for one diffusivity, numerous errors should be evaluated, displayed and stored. For a clear display of the composition-dependent diffusivities along the entire diffusion paths, and to save the space, only the average error of the diffusivities was provided here for simplification. This method has been successfully applied to evaluate the errors of the determined interdiffusivities in several ternary systems in our previous work $[13,14]$.

\section{Experimental procedure}

Aluminum (purity: 99.9 wt.\%), copper (purity: 99.99 wt.\%), and nickel (purity: 99.9 wt.\%) were used as starting materials. $\mathrm{Cu}-0.095 \mathrm{Al}$ and $\mathrm{Cu}-0.104 \mathrm{Ni}$ (both in mole fraction) were melted by arc melting under an Ar atmosphere using a non-consumable tungsten electrode (WKDHL-1, Opto-electronics Co., Ltd., Beijing, China) for four times to improve homogeneity. Subsequently, the two buttons were linearly cut into $5 \times 8 \times 1 \mathrm{~mm}^{3}$ after mechanically removing the surface material, and then sealed into evacuated quartz 
tubes, and homogenized at $1273 \mathrm{~K}$ for 20 days in a high-temperature furnace (GSL1700X, Hefei kejing materials technology Co., Ltd., Hefei, China), followed by water quenching. The polished and cleaned blocks from two alloys were bound together by molybdenum jig to form one diffusion couple. After that, the couple was sealed into an evacuated quartz tube, and annealed at $1273 \mathrm{~K}$ for $182 \mathrm{hrs}$, followed by quenching in cold water. The composition profiles of the polished diffusion couple were measured by means of EPMA technique (JXA-8230, JEOL, Japan). The error for concentration measurement is within $1 \%$.

\section{Results and discussion}

\section{Fictitious binary system}

To validate whether the pragmatic numerical inverse method is applicable in binary finite diffusion couple, a series of hypothetic composition profiles generated from four types of pre-assumed interdiffusivities were chosen as the target. By following the similar treatment in Ref. [21], one constant interdiffusion coefficient and three composition-dependent ones were pre-set, as summarized in Table 1 and also plot as the standard data in Fig. 2. The overall length for the binary diffusion couple was set to be $0.4 \mathrm{~mm}$. The number of mesh spacing along the $X$ direction was 200 and the mesh size was kept constant. The initial Matano plane was at the exact center of the $X$-axis. A geometry plot including boundary and initial conditions is displayed in Fig. 3. The generated composition profiles (denoted in symbol) of the finite diffusion couple, A$0.0001 \mathrm{~B} / \mathrm{B}-0.0001 \mathrm{~A}$ (in mole fraction), annealed at a certain temperature for $80 \mathrm{ks}$ are presented in Fig. 4. Considering that the real experimental data are always scattered, the Gaussian-distributed noise was added to the generated compositions of A and B components to mimic the scattered experimental data, as displayed in Fig. 5 (denoted by symbol).
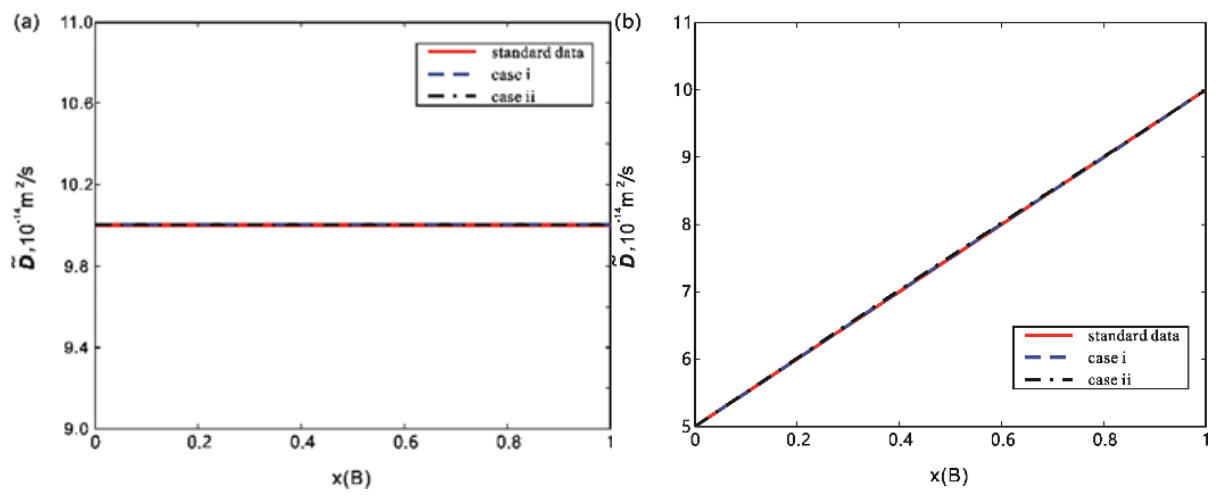

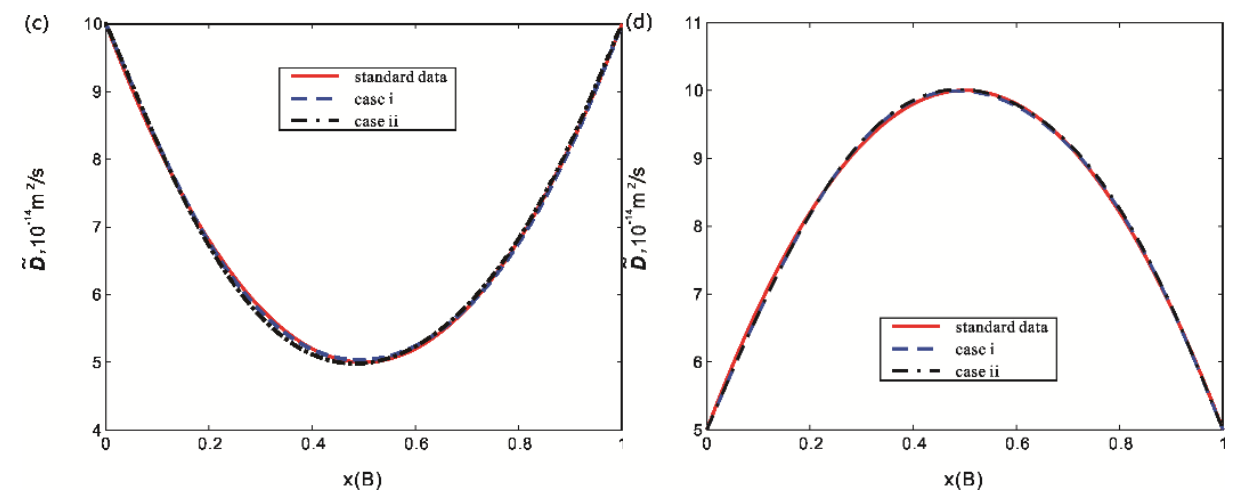

Fig. 2. Four types of pre-assumed interdiffusion coefficients curves (solid line) in comparison with the presently obtained results in Case $i$ and Case ii via the pragmatic numerical inverse method: (a) constant interdiffusion coefficient (b) linear composition dependence (c) quadratic composition dependence (d) quadratic composition dependence. $x_{B}$ is the mole fraction of component $B$.

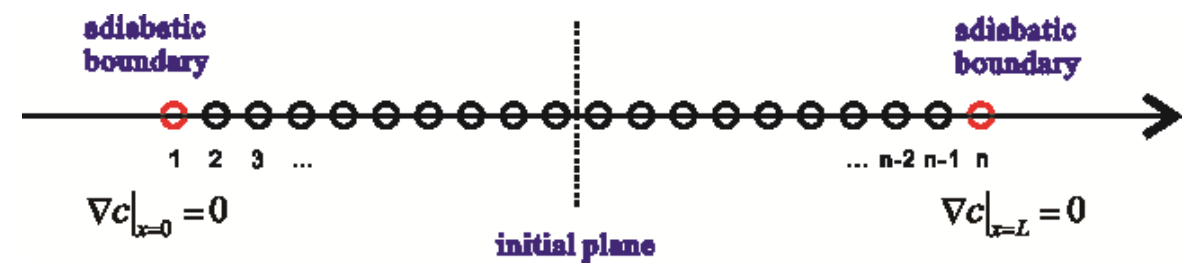

Fig. 3. A geometry plot including boundary and initial conditions for the fictitious binary A-B finite diffusion couple.
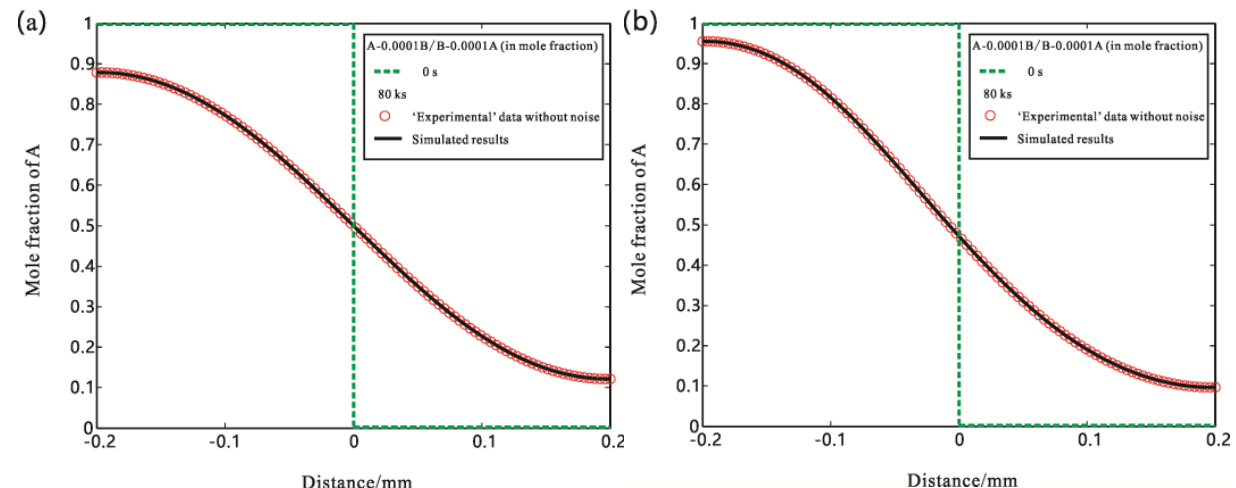

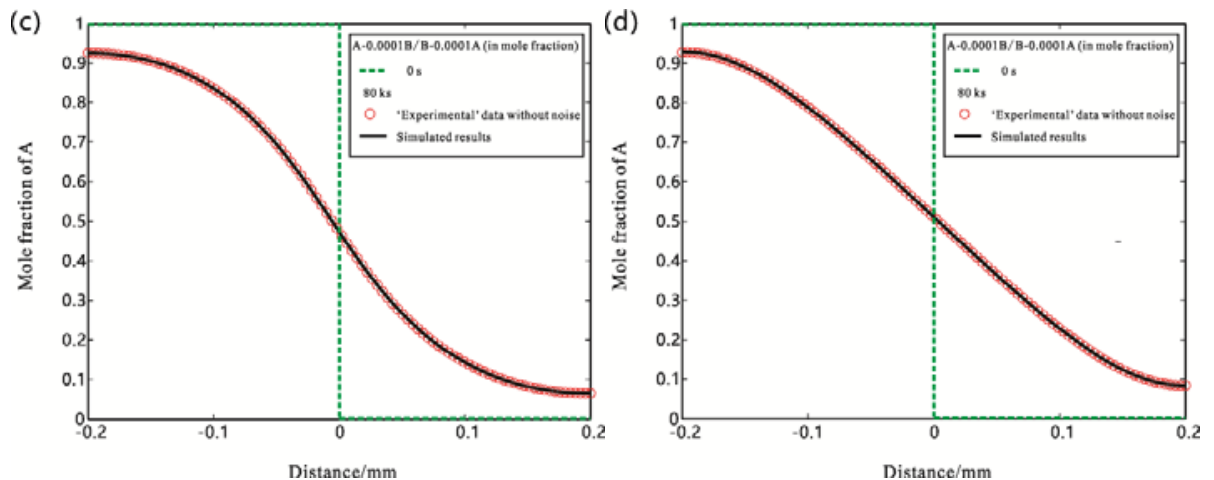

Fig. 4. Model-predicted composition profiles (denoted in solid line) for the diffusion couple $A-0.0001 \mathrm{~B} / \mathrm{B}-0.0001 \mathrm{~A}$ (in mole fraction) annealed for $80 \mathrm{ks}$ based on the evaluated interdiffusion coefficients, compared with those (denoted in symbols) generated based on the pre-assumed interdiffusion coefficients in Case i: (a) type 1 (b) type 2 (c) type 3 (d) type 4.
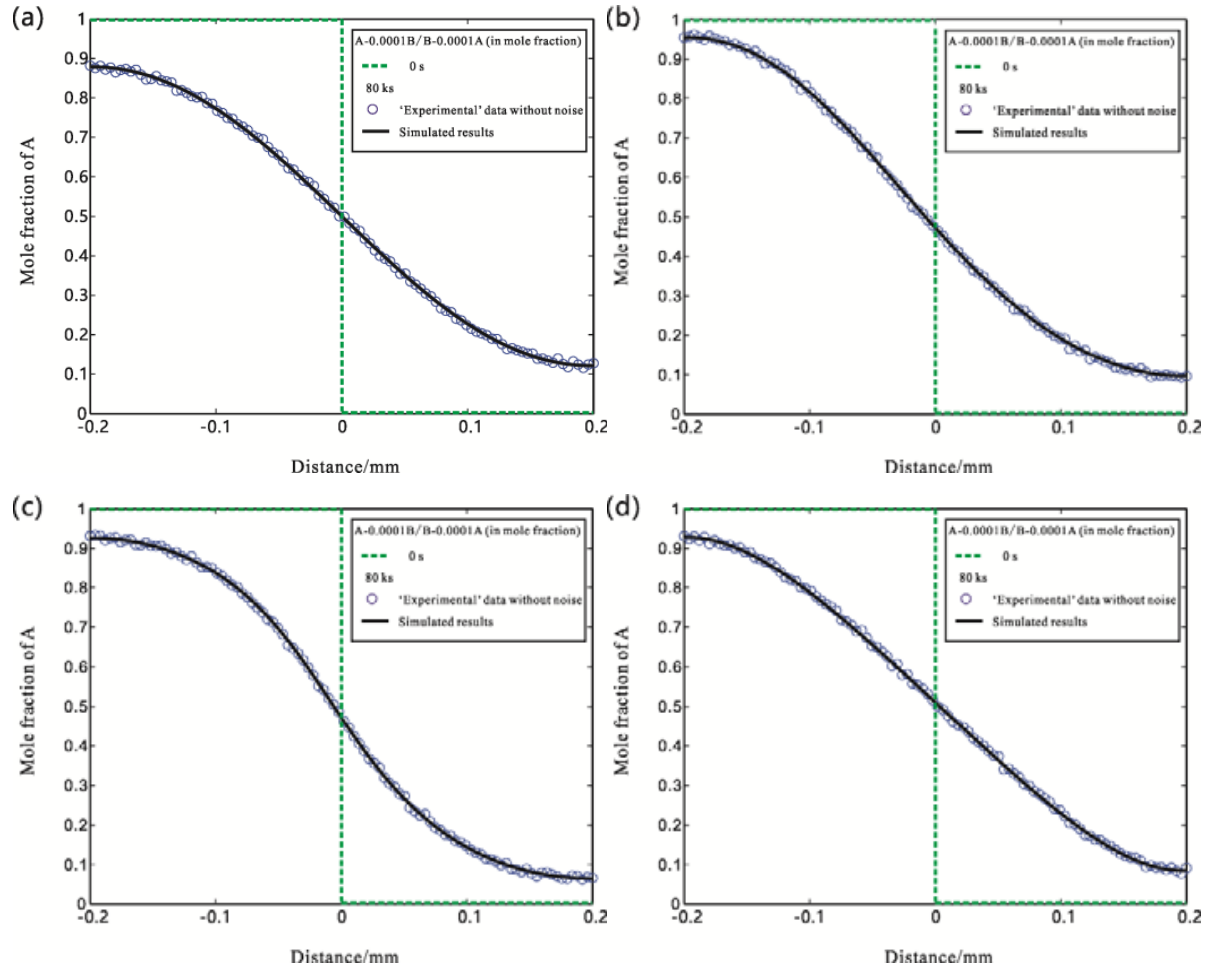

Fig. 5. Model-predicted composition profiles (denoted in solid line) for the diffusion couple $A-0.0001 \mathrm{~B} / \mathrm{B}-0.0001 \mathrm{~A}$ (in mole fraction) annealed for $80 \mathrm{ks}$ based on the evaluated interdiffusion coefficients, compared with those (denoted in symbols) generated based on the pre-assumed interdiffusion coefficients in Case ii: (a) type 1 (b) type 2 (c) type 3 (d) type 4. 
Since the A-B system is fictitious, a further assumption of thermodynamic description makes no sense. Here, the thermodynamic factor $\phi_{i}$ was simply set as 1 . It should be noted that end-members, $\Delta G_{A}^{B}$ and $\Delta G_{B}^{A}$, which correspond to the impurity diffusivities, can be first fixed according to the pre-assumed interdiffusion coefficients. Meanwhile, in order to decrease the number of adjustable parameters, it is assumed that $\Delta G_{A}^{A}=\Delta G_{B}^{A}$ and $\Delta G_{B}^{B}=\Delta G_{A}^{B}$ for simplification. The interaction parameters were set as adjustable parameters and optimized to achieve $\min \langle$ error $\rangle$ in Eq. (6). During the evaluation, the interaction parameters were introduced depending on whether they can really contribute to the minimization for the error between the measured and calculated composition profiles. Moreover, the vacancy flow effect shown in Eq. (2) was not taken into consideration in the present work.

Table 1. Four types of interdiffusion coefficients used in the present work.

\begin{tabular}{ccccc}
\hline \multicolumn{1}{c}{ constant } & $\begin{array}{c}\text { linear } \\
\text { composition } \\
\text { dependence }\end{array}$ & quadratic composition dependence \\
\hline No. & Type 1 & Type 2 & Type 3 & Type 4 \\
$\tilde{D} \times 10^{-13} m^{2} / s$ & 1 & $0.5 x_{B}+0.5$ & $2 x_{B}{ }^{2}-2 x_{B}+1$ & $-2 x_{B}{ }^{2}+2 x_{B}+0.5$
\end{tabular}

Note: $x_{B}$ is the mole fraction of component $B$

In order to test the applicability of the numerical inverse method in both ideal and real situations of the fictitious binary system, two cases were considered in the present work, i.e., Case $i$ is based on the composition profiles without noise, while Case $i i$ is with the composition profiles with the Gaussian-distributed noise (here, $0.5 \%$ standard deviation is set). For four types of interdiffusivities, different sets of adjustable parameters were chosen. For Type 1, no adjustable parameter was needed since the composition profiles can be fitted well by using only end-members. Two interaction parameters, $\Delta^{0} G_{A}^{A, B}$ and $\Delta^{0} G_{B}^{A, B}$, were chosen as adjustable parameters for Type 2. For Type 3, three interaction parameters, $\Delta^{0} G_{A}^{A, B}, \Delta^{0} G_{B}^{A, B}$ and $\Delta^{1} G_{B}^{A, B}$, were adopted. While for Type 4 , another three interaction parameters, $\Delta^{0} G_{A}^{A, B}, \Delta^{0} G_{B}^{A, B}$ and $\Delta^{1} G_{A}^{A, B}$, were taken into consideration.

The simulated composition profiles of the diffusion couple A-0.0001B/B-0.0001A (in mole fraction) annealed for $80 \mathrm{ks}$ were compared with the "experimental" data generated from the pre-assumed diffusivities in two cases, as shown in Figs. 4 and 5. As can be seen in both figures, the excellent agreement between the simulated composition profiles and the experimental data were achieved for all the four types of interdiffusivities. The evaluated interdiffusion coefficients by using the present numerical inverse in two cases are presented in Fig. 2, compared with the pre-assumed standard data. For Case $i$, i.e., when no noise was added to the composition profiles, the largest differences between 
the evaluated and the standard interdiffusion coefficients for four types are $0.02 \%, 0.08 \%$, $0.9 \%$ and $1.5 \%$, respectively. As for Case ii, i.e., when the Gaussian-distributed noise ( $\sigma$ $=0.5 \%)$ is added to the generated composition profiles, the evaluated interdiffusion coefficients show some difference from those in Case $i$, as indicated by Fig. 2. However, the largest differences between the evaluated and the standard interdiffusion coefficients for four types are $0.02 \%, 0.3 \%, 2.2 \%$ and $1.4 \%$ respectively, which are still acceptable. Consequently, all these facts validate the reliability of the present numerical inverse method in determination of the interdiffusivity for finite diffusion couple.

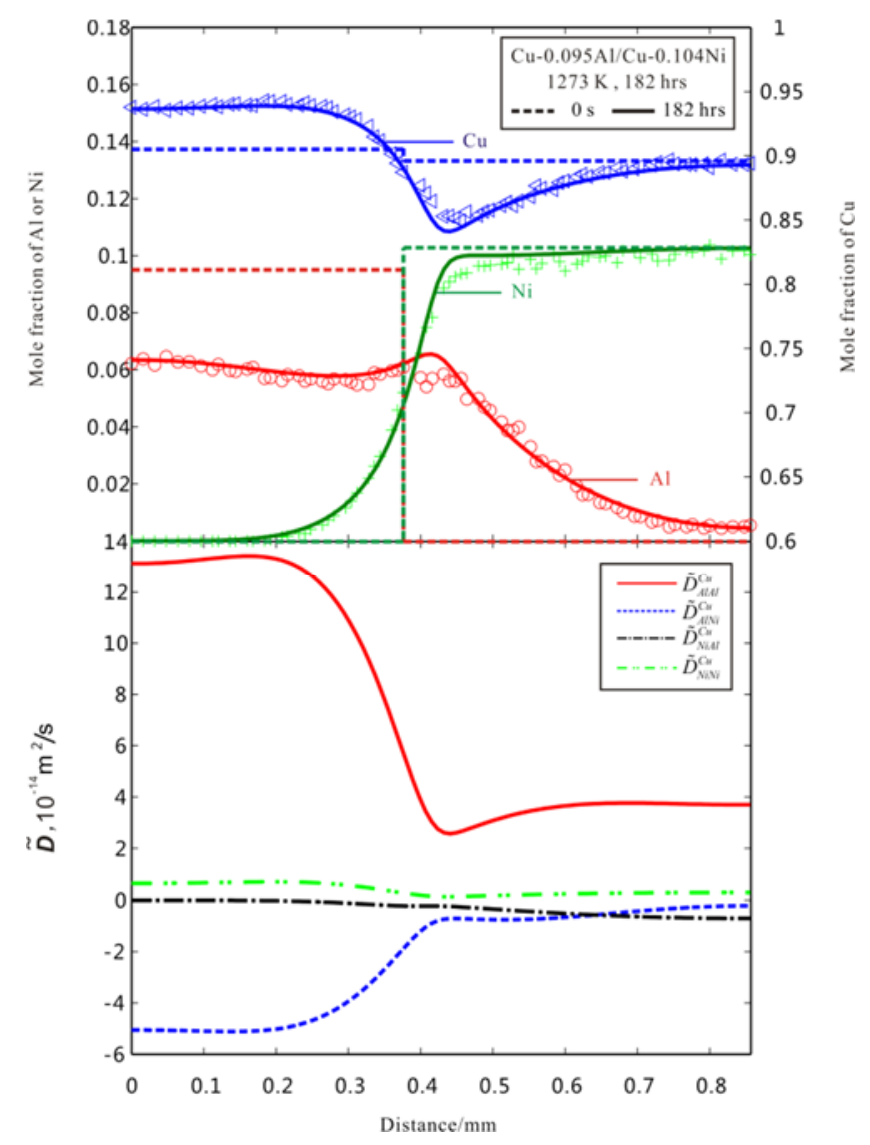

Fig. 6. Model-predicted composition profiles (denoted in solid lines) for the finite diffusion couple $\mathrm{Cu}-0.095 \mathrm{Al} / \mathrm{Cu}-0.104 \mathrm{Ni}$ (in mole fraction) annealed at $1273 \mathrm{~K}$ for

182 hrs based on the pragmatic numerical inverse approach, compared with experimental data (denoted by symbols). The corresponding interdiffusivities along the entire diffusion path obtained in the present work are also superimposed. 


\section{Al-Cu-Ni ternary system}

The composition profiles measured by EPMA for each component in $\mathrm{Cu}-$ $0.095 \mathrm{Al} / \mathrm{Cu}-0.104 \mathrm{Ni}$ (in mole fraction) diffusion couple annealed at $1273 \mathrm{~K}$ for $182 \mathrm{hrs}$ are presented in Fig. 6. It can be seen that the compositions of element $\mathrm{Al}$ at both ends of the diffusion couple deviate from the nominal ones, indicating that this diffusion couple falls into the finite type. Moreover, the uphill diffusion phenomenon can also be observed in the composition profile of component $\mathrm{Cu}$.

Based on composition profiles, the composition-dependent interdiffusivities of fcc $\mathrm{Al}-\mathrm{Cu}-\mathrm{Ni}$ diffusion couple at $1273 \mathrm{~K}$ were then determined by using the pragmatic numerical inverse method. During the evaluation, thermodynamic descriptions for the fcc A1 phase of the $\mathrm{Al}-\mathrm{Cu}-\mathrm{Ni}$ system [29] were taken into consideration and the endmembers for three binary systems were taken from Liu et al. [30]. All binary interaction parameters were regarded as adjustable. Meanwhile, the vacancy-wind effect was not taken into account in the present work. The thermodynamic descriptions and kinetic parameters used in the present work are listed in Table 2. With the final optimal set of adjustable parameters, the mole-fraction interdiffusivities [31] along the entire diffusion path can be obtained and are shown in Fig. 6. It should be noted that all the presently obtained interdiffusivities satisfy the thermodynamically stable constraints [24] and the average error for the obtained diffusivities was evaluated to be $9 \%$. In Fig. 6, the main interdiffusivities of component $\mathrm{Al}, \tilde{D}_{A l A l}^{C u}$, are greatly larger than those of component Ni, $\tilde{D}_{N i N i}^{C u}$, which is consistent with the experimental phenomenon that the fast diffusion of component $\mathrm{Al}$ makes the diffusion couple to be the finite type. In addition, the composition profiles were model-predicted by the computed interdiffusivities together with Fick's second law, and compared with the measured data, as also presented in Fig. 6. As can be seen in the plot, most of the experimental data can be well reproduced by the presently simulated composition profiles, except for slight deviations in the vicinity of the initial interface. The possible reasons lie in $i$ ) that it is very difficult to measure the compositions in the area close to the initial interface precisely using the EPMA technique considering the existence of the up-hill diffusion phenomena, and ii) that the case with up-hill diffusion phenomenon is always challenging for diffusivity evaluation. Though different sets of parameters were tried, no better fit can be obtained. Based on the above two aspects, in reality, it may be concluded that the fitting by using the present numerical inverse method is still very good for interdiffusivity evaluation, indicating the reliability of the present pragmatic numerical inverse method in ternary finite diffusion couples.

Table 2. Thermodynamic descriptions and kinetic parameters used in the present work.

\begin{tabular}{ccc}
\hline & Parameter & Value \\
\hline Thermodynamic descriptions & G(FCC_A1,AL,CU:VA;0) & $-53520.0+2.00 \times \mathrm{T}$ \\
& G(FCC_A1,AL,CU:VA;1) & $+38590.0-2.00 \times \mathrm{T}$ \\
& G(FCC_A1,AL,CU:VA;2) & +1170.0 \\
& G(FCC_A1,AL,NI:VA;0) & $-162407.8+16.21 \times \mathrm{T}$ \\
\hline
\end{tabular}




\begin{tabular}{|c|c|c|}
\hline & G(FCC_A1,AL,NI:VA;1) & $+73417.8-34.91 \times \mathrm{T}$ \\
\hline & G(FCC_A1,AL,NI:VA;2) & $+33471.0-9.84 \times \mathrm{T}$ \\
\hline & G(FCC_A1,AL,NI:VA;3) & $-30758.0+10.25 \times \mathrm{T}$ \\
\hline & G(FCC_A1,CU,NI:VA;0) & $+8047.7+3.42 \times \mathrm{T}$ \\
\hline & G(FCC_A1,CU,NI:VA;1) & $-2041.3+1.00 \times \mathrm{T}$ \\
\hline & G(FCC_A1,AL,CU,NI:VA;0) & -100000.0 \\
\hline & G(FCC_A1,AL,CU,NI:VA;0) & $-120000.0-50.00 \times \mathrm{T}$ \\
\hline \multirow[t]{9}{*}{ End-members } & $\Delta G_{A l}^{A l}$ & $-123111.6-97.34 \times \mathrm{T}$ \\
\hline & $\Delta G_{A l}^{C u}$ & $-181583.4-99.8 \times \mathrm{T}$ \\
\hline & $\Delta G_{A l}^{N i}$ & $-268381.0-71.04 \times \mathrm{T}$ \\
\hline & $\Delta G_{C u}^{A l}$ & $-133184.4-83.65 \times \mathrm{T}$ \\
\hline & $\Delta G_{C u}^{C u}$ & $-205872.0-82.52 \times \mathrm{T}$ \\
\hline & $\Delta G_{C u}^{N i}$ & $-263689.7-77.04 \times \mathrm{T}$ \\
\hline & $\Delta G_{N i}^{A l}$ & $-144600.0-64.848 \times \mathrm{T}$ \\
\hline & $\Delta G_{N i}^{C u}$ & $-229936.8-72.83 \times \mathrm{T}$ \\
\hline & $\Delta G_{N i}^{N i}$ & $-271377.6-81.79 \times \mathrm{T}$ \\
\hline \multirow[t]{5}{*}{ Adjustable parameters } & $\Delta G_{A l}^{A l C u}$ & -329369.0 \\
\hline & $\Delta G_{A l}^{A l N i}$ & -418941.0 \\
\hline & $\Delta G_{C u}^{A l C u}$ & -227489.0 \\
\hline & $\Delta G_{C u}^{C u N i}$ & -222173.0 \\
\hline & $\Delta G_{N i}^{A l N i}$ & -474088.0 \\
\hline
\end{tabular}




\begin{tabular}{cc}
\hline$\Delta G_{N i}^{C u N i}$ & -293795.0 \\
$\Delta G_{A l}^{C u N i}$ & -150253.0 \\
$\Delta G_{N i}^{A l C u}$ & -485865.0 \\
$\Delta G_{C u}^{A l N i}$ & -808646.0
\end{tabular}

\section{Summary}

Considering that the traditional Boltzmann-Matano method and its variants have a complex process for calculation of interdiffusion coefficients in finite diffusion couples, especially for ternary and higher-order systems or the cases with up-hill diffusion phenomenon, the previously developed pragmatic numerical inverse method was adopted to determine the composition-dependent interdiffusion coefficients in single-phase finite diffusion couples. In a fictitious A-B system, four different types of binary interdiffusion coefficients were assumed as standard data to generate the fictitious composition profiles in a binary finite diffusion couple, A-0.0001B/B-0.0001 A (in mole fraction) annealed for $80 \mathrm{ks}$. In order to mimic the true "experimental" data, the Gaussian-distributed noise was added in. Based on the composition profiles generated from the pre-assumed standard interdiffusivity data, the interdiffusion coefficients were determined by using the pragmatic numerical inverse method and found to be nicely consistent with the standard interdiffusion coefficients. Moreover, the numerical inverse method was then further applied in a real ternary finite diffusion couple, $\mathrm{Cu}-0.095 \mathrm{Al} / \mathrm{Cu}-0.104 \mathrm{Ni}$ (in mole fraction), annealed at $1273 \mathrm{~K}$ for $182 \mathrm{hrs}$. Based on the composition profiles measured by EPMA, ternary interdiffusion coefficients along the entire diffusion path of this ternary diffusion couple were obtained by the pragmatic numerical inverse approach. Furthermore, the measured composition profiles can be well reproduced by the modelpredicted results.

\section{Acknowledgments}

The financial support from the National Key Research and Development Program of China (Grant No. 2016YFB0301101), the National Natural Science Foundation of China (Grant No. 51474239), and the Hunan Provincial Natural Science Foundation for Youth of China (Grant No. 2015JJ3146) is acknowledged. 


\section{References}

[1] A. Engström, J.E. Morral, J. Ágren: Acta Mater, 45 (1997) 1189-1199.

[2] L. Zhang, Y. Du, Y. Ouyang, H. Xu, X. Lu, Y. Liu, Y. Kong, J. Wang: Acta Mater, 56 (2008) 3940-3950.

[3] C. Matano: Jpn J Phys, 8 (1933) 109-113.

[4] F. Sauer, V. Freise: Z Elektrochem, 66 (1962) 353-362.

[5] C. Wagner: Acta Metall, 17 (1969) 99-107.

[6] J.S. Kirkaldy: Can J Phys, 35 (1957) 435-440.

[7] M. Liu, L. Zhang, W. Chen, J. Xin, Y. Du, H. Xu: CALPHAD, 41 (2013) 108-118.

[8] J. Chen, C. Zhang, J. Wang, W. Chen, Y. Tang, L. Zhang, Y. Du: CALPHAD, 50 (2015) 118-125.

[9] J. Chen, J. Xiao, L. Zhang, Y. Du: J Alloys Compd, 657 (2016) 457-463.

[10] J. Li, W. Chen, D. Liu, W. Sun, L. Zhang, Y. Du, H. Xu: J Phase Equilib Diffus, 34 (2013) 484-492.

[11]J. Li, T. Liu, W. Chen, S. Wang, L. Zhang, Y. Du: J Min Metall Sect B-Metall, 50 (2014) 93-99.

[12]R. Wang, L. Zhang, Z. Lu, Y. Du, Z. Jin, D. Živković: J Phase Equilib Diffus, 36 (2015) 510-517.

[13] W. Chen, L. Zhang, Y. Du, C. Tang, B. Huang: Scripta Mater, 90-91 (2014) 53-56.

[14]H. Xu, W. Chen, L. Zhang, Y. Du, C. Tang: J Alloys Compd, 644 (2015) 687-693.

[15] J. Chen, L. Zhang, J. Zhong, W. Chen, Y. Du: J Alloys Compd, 688 (2016) 320-328.

[16] Y. Liu, W. Chen, Y. Tang, Y. Du, L. Zhang: Acta Metall Sin, 52 (2016) 1009-1016.

[17] S. Deng, W. Chen, J. Zhong, L. Zhang, Y. Du, L. Chen: CALPHAD, 56 (2017) 230240.

[18] S. Wen, Y. Tang, J. Zhong, L. Zhang, Y. Du, F. Zheng: J Mater Res, 32 (2017) 21882201.

[19]W. Chen, J. Zhong, L. Zhang: MRS Commun, 6 (2016) 295-300.

[20] W. Chen, L. Zhang: J Phase Equilib Diffus, 38 (2017) 457-465.

[21]T. Ahmed, I.V. Belova, A.V. Evteev, E.V. Levchnko, G.E. Murch: J Phase Equilib Diffus, 36 (2015) 366-374.

[22] J.R. Manning: Metall Trans, 1 (1970) 499-505.

[23] J.O. Andersson, J. Ágren: J Appl Phys, 72 (1992) 1350-1355.

[24]L. Onsager: Ann N Y Acad Sci, 46 (1945) 241-265.

[25]A.V. Jaques, J.C. LaCombe: J Phase Equilib Diffus, 33 (2012) 181-188.

[26] J.E. Morral, W.D. Hopfe: J Phase Equilib Diffus, 35 (2014) 666-669.

[27] W.D. Hopfe, J.E. Morral: J Phase Equilib Diffus, 37 (2016) 110-118.

[28] J. Léchelle, S. Noyau, L. Aufore, A. Arredondo, F. Audubert: Diffus Fundam Org, 17 (2012) 1-39.

[29] Y. Du, S. Liu, L. Zhang, H. Xu, D. Zhao, A. Wang, L. Zhou: CALPHAD, 35 (2011) 427-445.

[30]D. Liu, L. Zhang, Y. Du, S. Cui, W. Jie, Z. Jin: Int J Mater Res, 104 (2013) 135-148.

[31]J. E. Morral: J Phase Equilib Diffus, 35 (2014) 581-586.

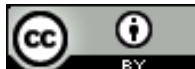

Creative Commons License

This work is licensed under a Creative Commons Attribution 4.0 International License. 